\title{
Der Bildungsauftrag im Schweizer Kinderfernsehen
}

\author{
Ursula Schwarb, Sara Signer und Heinz Bonfadelli
}

\section{Einleitung}

Im Rahmen des vom Bundesamt für Kommunikation finanzierten Projektes Öffentlicher Rundfunk und Bildung. Angebot, Nutzung und Funktion von Kinderprogrammen wurde erstmals die Entwicklung des Kinderfernsehens wie auch des Kinderradios in der Schweiz umfassend nachgezeichnet und in ein Verhältnis zum in der schweizerischen Bundesverfassung formulierten Bildungsauftrag gesetzt. Im Zentrum der Studie stehen folgende Fragen: Wie hat sich das Bildungsangebot der Schweizerischen Rundfunkgesellschaft SRG in der Deutschen Schweiz für das Segment der Kinder entwickelt? Wie werden die Leistungen der SRG für den Bereich «bildende Kinderangebote» umgesetzt und beurteilt? Zur Beantwortung der Fragestellungen wurde das Fernseh- und Radioangebot für Kinder im Zeitraum von 1980-2006 sowie die zugehörigen Homepages (Stand 2005/06) inhaltsanalytisch untersucht. Zudem wurden eine Dokumentenanalyse und eine Expertenbefragung mit insgesamt 22 gegenwärtigen und früheren Machern/-innen sowie externen Beobachtern/-innen durchgeführt. Die Studie wurde Ende Februar 2007 abgeschlossen. Der vorliegende Beitrag konzentriert sich ausschliesslich auf das Kinderfernsehen. Bevor die Ergebnisse der Fernsehprogrammanalyse sowie die Ergebnisse der Expertenbefragung dargestellt werden, soll kurz auf die Ausgangslage «Kinderfernsehen in der Schweiz» eingegangen werden.

\subsection{Die Anfänge des Kinderfernsehens in der Schweiz}

Vergleichbar zu anderen europäischen Ländern liegen die Anfänge des Kinderfernsehens in der Schweiz in den 1950er-Jahren. Als eigentlicher Meilenstein gilt allerdings das Jahr 1968, in welchem dem Schweizer Fernsehen eine eigentliche Pionierleistung gelang, indem es als erster Sender im deutschsprachigen Raum ein wöchentliches Vorschulprogramm Spielhaus ausstrahlte. Dies geschah anfänglich in enger Kooperation mit der englischen BBC, welche in diesem Programmbereich zu den führenden Anbietern zählte. Ebenfalls 1968 begann die lange und bis heute anhaltende Serie der Gutenacht-Geschichten. Die programmlichen Investitionen und Innovationen für Kinder waren auf verschiedene Faktoren zurückzuführen: Einerseits bedeutete die Gründung der Abteilung «Familie und Erziehung» (später Familie und Bildung) im Jahre 1964 eine starke institutionelle Abstützung der 
Programmaktivitäten im Bereich Kinder und Bildung. Andererseits herrschte damals im Schweizer Fernsehen eine programmpolitische Aufbruchstimmung. Die SRG sah sich 1969 durchaus als Teil des Bildungssystems und betrachtete als ihre Aufgabe,

ein anregendes Milieu für Kinder im Vorschulalter zu schaffen, das heisst im Einzelnen, die intellektuellen Verfahrensweisen zu verbessern, die Beweggründe für Leistung zu vermitteln und die Sprache gezielt zu fördern (Portmann 1969: 31).

Das Wirkungspotential, das dem Fernsehen als Bildungsmedium zugeschrieben wurde, war wissenschaftlich abgestützt und reflektiert (u. a. 1976-1979 medienpsychologische Untersuchung von SF-Kinderprogrammen hinsichtlich Verständnis, Aufmerksamkeit, Aktivierung etc. durch Hertha Sturm). So kam damals aus der Entwicklungspsychologie die Erkenntnis, dass Kinder im Vorschulalter ausserordentlich lernfähig sind und dieses Alter eine optimale Zeit für die Begabungsförderung darstellt. Zudem ging man davon aus, dass das Fernsehen Bildungschancen ausgleichen könne. Auch wenn sich alsbald verschiedene der gehegten Erwartungen nicht erfüllten, investierte das SF bis in die späten 1980er Jahre hinein in sein Kinderprogramm. Ziele waren kreative Aktivierung, Partizipation, Emanzipation und Integration als soziales Lernen (vgl. z. B. Doelker 1979). Auf Ebene der Programme wurden neben Spielhaus Kinder- und Jugendsendungen verschiedenster Art, z. T. mit renommierten Künstlern und mit edukativem, pädagogischem oder medienerzieherischem Impetus, selber konzipiert oder (co-) produziert (Franz und René, Zeichnen mit Scapa, 1, 2 oder 3, Was man weiss und doch nicht kennt, Ach so ist das!, Mattscheibe - Klarscheibe etc.). Auf dem Hintergrund dieser Tradition der pädagogisch fundierten und am Bildungsauftrag orientierten Kinderprogramme interessiert, wie sich das Bildungsangebot im Schweizer Fernsehen für das Segment der Kinder weiterentwickelt hat und wo es heute steht.

\section{Programmanalyse des Schweizer Kinderfernsehens}

2.1 Fragestellung und Methode

Wie entwickelte sich das Kinderprogramm im Schweizer Fernsehen seit 1980 und wie gross ist das Bildungspotential des Schweizer Kinderprogramms heute? Diese Fragen waren für die Programmanalyse wegleitend und wurden mit den folgende Untersuchungsdimensionen angegangen: 1. formale Aspekte der Kindersendungen, 2. Sendeformate und Genres, 3. Bildung und Unterhaltung, 4. Interaktivität, 5. Themengebiete und 6 . Zielgruppenangaben.

$\mathrm{Da}$ auf keine Originalsendungen zurückgegriffen werden konnte, wurde die Programmanalyse als Längsschnittanalyse auf der Basis der Fernsehzeitschrift «Tele» 
durchgeführt, wobei für das letzte Jahr (2006) auch Onlinefernsehzeitschriften beigezogen wurden. Untersucht wurde jeweils die erste Märzwoche der Jahre 1980, 1983, 1985, 1988, 1990, 1993, 1995, 1998, 2000, 2003 und 2006, wobei analog zur Bestandsaufnahme des Kinderfernsehens von Bachmair der Dienstag, Samstag und Sonntag ausgewählt wurden (vgl. Bachmair 1996: o.S.). Mit der fünffachen Gewichtung des Dienstags wurde eine künstliche Woche gebildet. Wie im Titel des BAKOM-Projektes Öffentlicher Rundfunk und Bildung. Angebot, Nutzung und Funktionen von Kinderprogrammen bereits klar zum Ausdruck kommt, werden die öffentlich-rechtlichen Sender SF1 und SF2 untersucht, welche auch gesetzlich ${ }^{1}$ einem Bildungsauftrag verpflichtet sind. Analysiert wurden alle Sendungen zu den oben genannten Zeitpunkten, die explizit als Kinderprogramm in der Programmzeitschrift ausgewiesen wurden oder anhand von Sendebeschreibungen als solche identifiziert werden konnten. Die Sendebeschreibungen haben für die Codierung des Bildungspotentials eine wesentliche Bedeutung, da auf der Ebene der Programmzeitschrift das Bildungspotential nicht erfasst werden kann.

Tabelle 1: Anzahl untersuchter Kindersendungen

\begin{tabular}{|l|r|r|r|r|r|r|r|r|r|r|r|r|}
\hline Jahr & \multirow{2}{*}{1980} & 1983 & 1985 & 1988 & 1990 & 1993 & 1995 & 1998 & 2000 & 2003 & 2006 & Total \\
\hline Sender & 19 & 18 & 18 & 19 & 18 & 28 & 18 & 12 & 22 & 18 & 2 & 192 \\
\hline SF 2 & & & & & & & & 24 & 82 & 110 & 180 & 396 \\
\hline SF1 und SF2 & 19 & 18 & 18 & 19 & 18 & 28 & 18 & 36 & 104 & 128 & 182 & 588 \\
\hline
\end{tabular}

\subsection{Operationalisierung des Bildungspotentials}

Wie Tabelle 1 zeigt, kann im Folgenden, nach der fünffachen Gewichtung des Dienstags und ohne Schulfernsehsendungen, mit einer Datenbasis von 588 Kindersendungen gearbeitet werden, wobei 196 Kindersendungen codiert wurden.

Wer mit Programmkategorien arbeitet, dem ist bekannt, dass abstrakte Programmbezeichnungen wie z. B. «Bildung», «Unterhaltung» oder «Information» an ihren Grenzen unscharf sind. Will man aber nicht darauf verzichten, sind schon auf Sendungsebene Konventionen erforderlich, welche Sendung wie behandelt werden soll, damit eine reliable begriffliche Währung garantiert werden kann (vgl. Krüger 2001: 74). Problematisch ist bei Begriffen wie «Information» und «Unterhaltung», aber auch «Bildung», dass sie implizit mit normativen Vorstellungen verbunden sind und somit besonders darauf geachtet werden muss, dass mit wissenschaftlicher Neutralität gearbeitet wird (vgl. Hohlfeld 1998: 198). 
Wie kann nun angesichts solcher Schwierigkeiten das Bildungspotential einer Kindersendung zuverlässig bestimmt werden? - Grundsätzlich ist davon auszugehen, dass es sich nicht nur um entweder bildende oder nicht bildende Sendungen handelt, sondern sendungsbezogen eine Abstufung unterschiedlicher Bildungspotentiale existiert. Aus diesem Grund wurde eine Kategorisierung entwickelt, welche eine Abstufung des Bildungspotentials von Kindersendungen zulässt.

Ebenfalls zu berücksichtigen ist, dass es sich bei «Bildung» resp. "Information» und "Unterhaltung» nicht um Gegensätze handelt, sondern um Extrempositionen, welche einen sehr breiten Graubereich der Durchmischung zulassen. Die bekannteste Mischung ist wohl das sogenannte «Infotainment».

Vor dem Hintergrund der Annahme, dass es kaum reine Programmformate gibt, lauten die Codieranweisungen folgendermassen:

- Schulfernsehen: von der Programmzeitschrift explizit als Schulfernsehen ausgewiesen.

- Dominant bildend, aber nicht Schulfernsehen: Sendungen, die nicht explizit als Bildungssendung ausgewiesen sind, deren primäres Ziel aber die Vermittlung von lehrreichen, bildenden Inhalten ist. Beispiel: Spielhaus

- Implizit bildend und Infotainment: Sendungen, die bestimmte Elemente bzw. Themen enthalten, die als lehrreich, bildend eingestuft werden können. Solche bildenden Elemente sind meist eingebettet in eine oder mehrere unterhaltende (Rahmen-)Handlungen oder werden selbst unterhaltend präsentiert. Von besonderer Bedeutung sind Themen, welche entscheidend sind für die soziale Interaktion und Integration. Der Sendung kann insgesamt eine potentielle Bildungsfunktion zugeschrieben werden. Beispiele: Lazy Town, Trashup, Live.

- Nicht bildend, unterhaltend: Sendungen, in denen Unterhaltung dominiert. Es sind keine bzw. kaum bildende Elemente vorhanden. Beispiele: Catdog, Cosmo und Wanda.

\subsection{Ergebnisse Programmanalyse des Schweizer Kinderfernsehens 1980-2006}

Im Folgenden können nicht alle Ergebnisse der Studie präsentiert werden, sondern eine Auswahl besonders interessanter Aspekte. Zu den einzelnen Dimensionen werden als erstes die gewonnen Ergebnisse dargestellt und anschliessend, falls möglich, mit deutschen Studien verglichen. Hierbei relevant sind die Studien von Krüger u.a. (1995-2007), Trebbe/Weiss (1998-1999) und Bachmair/Lambrecht (2000-2003.). Die Studien von Krüger u. a. fokussieren bei ihrer Programmanalyse nicht speziell auf das Kinderprogramm, dieses wird aber, wie auch bei Weiss und Trebbe (2000) als eine der Hauptkategorien erfasst Im Gegensatz dazu konzentrieren sich Bachmair und Lambrecht im Rahmen der 
«Bestandsaufnahme zum Kinderfernsehen» speziell auf das Kinderangebot in der Deutschen Fernsehlandschaft.

\subsubsection{Anzahl der SF-Kindersendungen 1980-2006}

Grafik 1 zeigt deutlich, dass die Anzahl der Kindersendungen insgesamt auf SF1 und SF2 zugenommen hat. Da ab dem Jahr 1997 der zweite Schweizer Sender (SF zwei) seinen Betrieb aufnimmt und inhaltlich auf Kinder und Sport ausgerichtet ist, sinkt die Anzahl an Kinderprogrammen auf SF1 ab diesem Zeitpunkt bis heute weiter ab, nimmt aber auf SF zwei enorm zu.

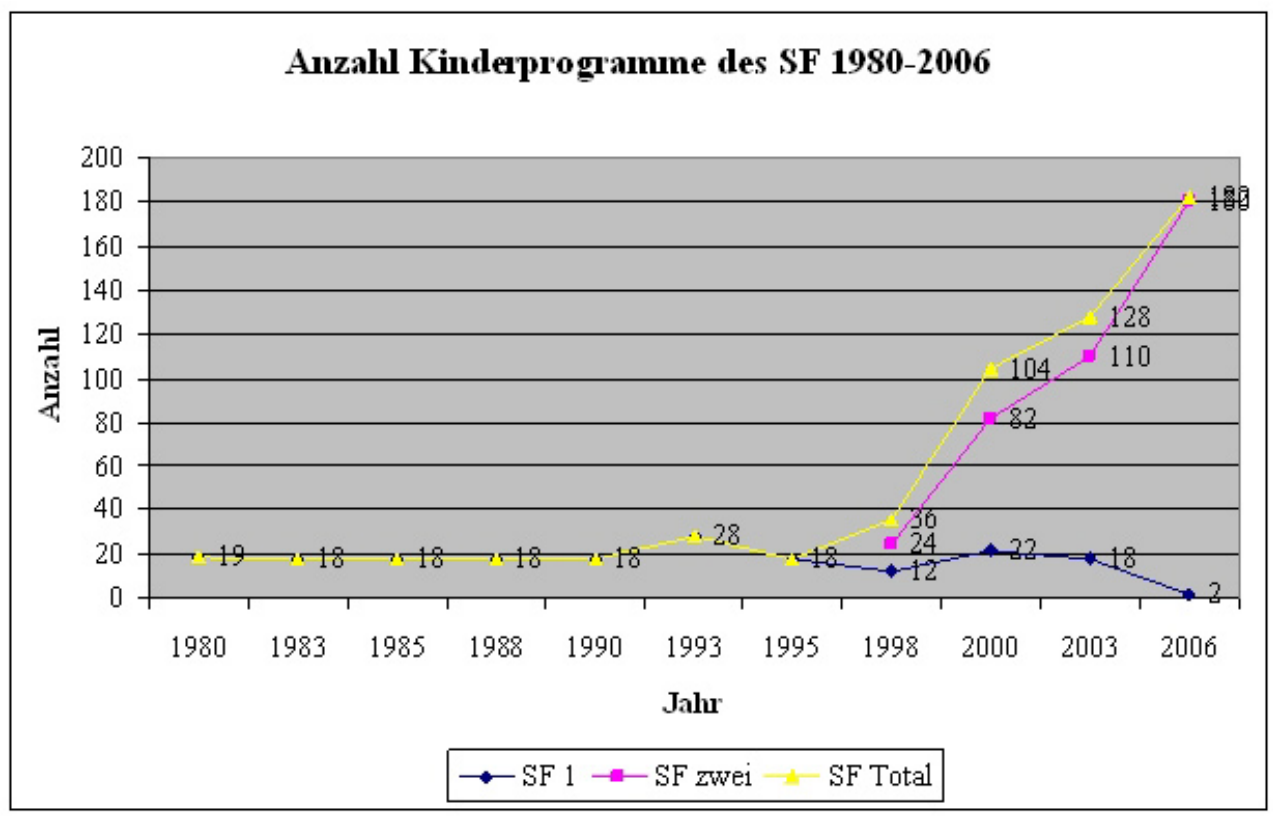

Grafik 1: Wachstum der SF-Kindersendungen 1980-2006 $(n=588)$

\subsubsection{Prozentualer Anteil der Kindersendungen am Gesamtprogramm}

Doch wie verhält sich die Zahl der Kindersendungen zum Gesamtprogramm? Tabelle 2 zeigt deutlich, dass im Verhältnis zum Gesamtprogramm der Anteil des Kinderprogramms nicht zugenommen hat, wie man aus Grafik 1 schliessen könnte, sondern abnahm. Der Anteil des Kindersendungen am Gesamtprogramm sank bei SF2 gar von 6.1\% im Jahr 2000 auf 3.5\% im Jahr 2005. 
Tabelle 2: Zeitlicher Anteil der Kindersendungen beim SF 1976-2005

\begin{tabular}{|l|r|r|r|r|r|r|r|}
\hline Angaben in \% & 1976 & 1980 & 1985 & 1990 & 1995 & 2000 & 2005 \\
\hline SF DRS / SF & 7.4 & 8.2 & 5.6 & 5.5 & 5.7 & 4.7 & 3.2 \\
\hline SF 1 & & & & & & 1.4 & 0.3 \\
\hline SF zwei & & & & & & 6.1 & 3.5 \\
\hline
\end{tabular}

Anmerkung zu Tabelle 2: Anteil Kinderprogramm bei SF DRS aufgrund der SRG-Programmstatistiken, ohne B bzw. B3, d. h. Werbung, Trailer, Füller, Wetterkarten; für $2000+2005$ Anteile addiert.

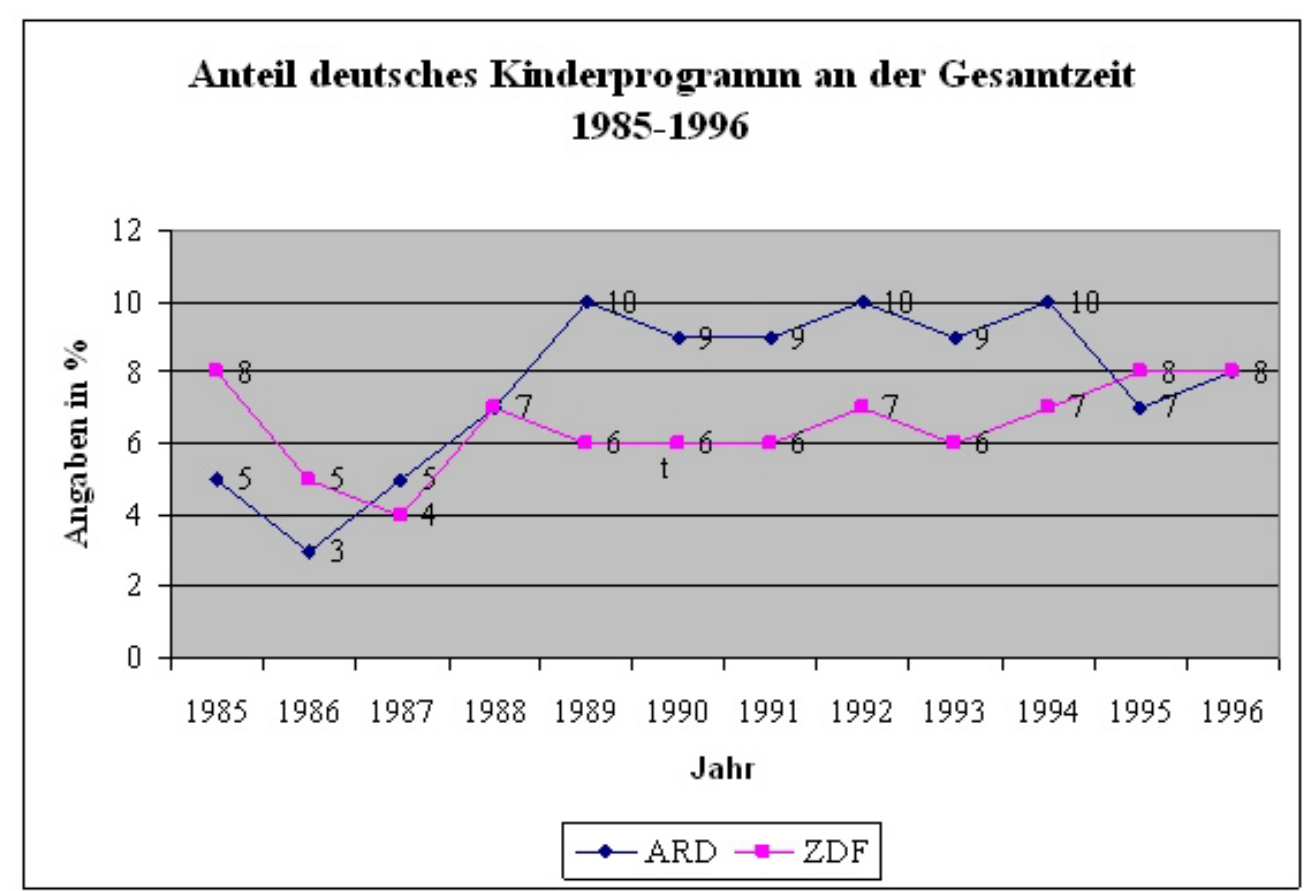

Grafik 2: Entwicklung des Kinderprogramms an der Gesamtsendezeit bei ARD und ZDF (Quellen: Krüger 1996a: 421; Krüger 1996b: 359)

Das SF-Kinderprogramm erreichte seinen Höhepunkt hinsichtlich des zeitlichen Umfangs Anfang der 1980er Jahre. Zu diesem Zeitpunkt waren zwei verschiedene Haltungen gegenüber dem Kinderfernsehen in der Öffentlichkeit deutlich zu spüren, welche aber im Endeffekt beide den hohen Anteil an Kinderprogrammen erklären oder zumindest miterklären können: Auf der einen Seite sollten Kinderprogramme edukativ aufbereitet sein und sollten auch in die Schule integriert werden (vgl. Stalder 1978:2; Loretz 1979:3), auf der anderen Seite stand die Privatisierung vor der Tür und mit ihr die Angst vor der neuen privaten Konkurrenz.

Bis Mitte der 1990er Jahre sinkt die Anzahl Kindersendungen wieder deutlich $a b$, was mit der institutionellen Schwächung des Kinderprogramms aufgrund 
der Auflösung der Abteilung «Familie und Bildung» parallel geht. Zwar steigt auf das Jahr 2000 hin das Angebot an Kindersendungen im Verhältnis zum restlichen Programm wieder stark an, sinkt aber bereits 2005 auf einen vorher nicht da gewesenen Tiefstand.

Eine andere Entwicklung zeigt sich hingegen bei den öffentlichen Rundfunkanbietern in Deutschland (siehe Grafik 2). Krügers Programmanalysen dokumentieren eine Ausweitung des Kinderprogramms bei ARD und ZDF nach der Dualisierung, wobei hier der Kinderkanal keine Berücksichtigung findet.

\subsubsection{Darstellungsformen von SF-Kindersendungen 1980-2006}

Wie Kindersendungen produziert werden und in welcher Darstellungsform diese schliesslich ausgestrahlt werden, hängt zum einen mit der technischen Entwicklung und zum anderen mit den Produktionskosten zusammen. Deutlich zeigt Grafik 3, dass die realen Personen schon immer in Kindersendungen vorkamen, im Gegensatz zum Puppenspiel oder dem Knettrick, welche beide über den gesamten Zeitraum hinweg nur vereinzelt vertreten waren. Explosionsartig zugenommen hat hingegen das Angebot an Zeichentricksendungen seit Mitte der 1990er Jahre, was unter anderem mit ihrem eher günstigen Einkaufspreis im Unterschied zu teuren Eigenproduktionen erklärt werden kann. Zudem zeigt sich deutlich, dass die Gesamtzunahme des Kinderprogramms speziell auf die Trickfilme zurückzuführen ist.

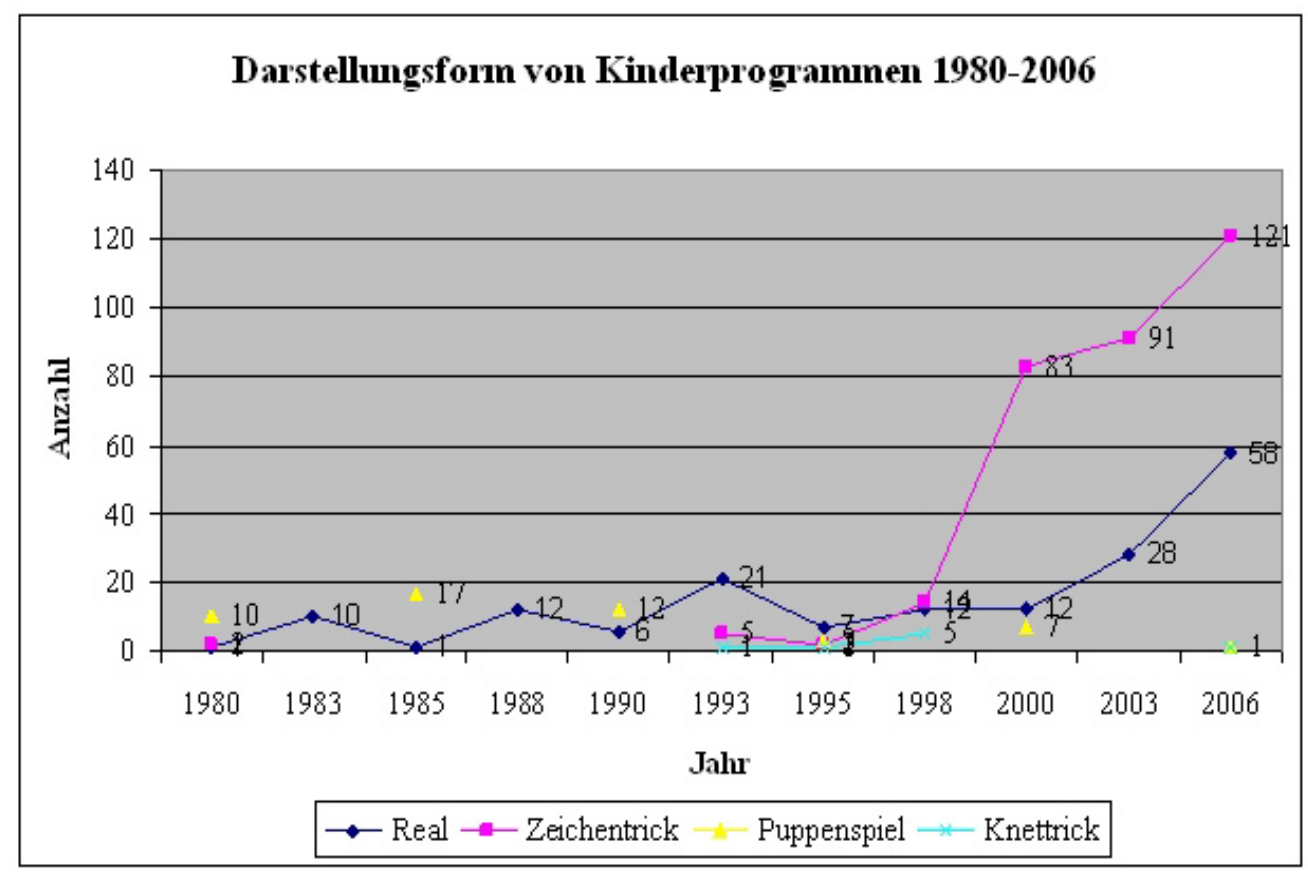

Grafik 3: Darstellungsformen von SF-Kindersendungen 1980-2006 ( $n=583)$ 
Auch Lambrecht/Bachmair haben in ihrer Untersuchung "Bestandsaufnahme zum Kinderfernsehen 2000» die Darstellungsweisen des deutschen Kinderfernsehens untersucht. Sie unterschieden folgende Kategorien: "Realfilm», "Animation ohne Puppenspiel» und «Puppenspiel». Für das Jahr 2003 zeigt sich bei den öffentlich-rechtlichen deutschen Sendern die Verteilung folgendermassen: 45 Prozent Realfilm, 36 Prozent Animation, 19 Prozent Puppenspiel (vgl. Lambrecht 2002: 9). Ein Vergleich mit dem SF zeigt ein deutliches Übergewicht an Zeichentrickdarstellungen im Schweizer Fernsehen, was im deutschen Kinderfernsehen eher dem Angebot der Privatsender entsprechen würde.

«Inhaltlich ist Kinderfernsehen in Deutschland - daran hat sich nichts geändert und wird sich wohl auch nichts ändern - vorwiegend Kinderunterhaltung, die vor allem in Form von Zeichntrickserien präsentiert wird» (vgl. Weiss 2000: 72 zit. nach Gangloff). Eine ähnliche Situation zeigt sich in der Schweiz.

\subsubsection{Produktionsland der SF-Kindersendungen 1980-2006}

Bis Mitte der 90er Jahr bleibt der Anteil an eigenproduzierten Kindersendungen stabil (siehe Grafik 4), fällt 1998 auf seinen vorläufigen Tiefpunkt und steigt mit der allgemeinen Zunahme an Kinderprogrammen wieder stark an. Die deutschen Produktionen verhalten sich in ihrer Tendenz ähnlich, allerdings insgesamt auf einem tieferen Niveau an Eigenproduktionen.

Zwischen 1998 und 2003 kommt es zu einem enormen Zuwachs an amerikanischen Produkten. Dieser Trend scheint heute wieder etwas abzuflauen, ist aber gleichzeitig verbunden mit einer Zunahme an asiatischen Kindersendungen.

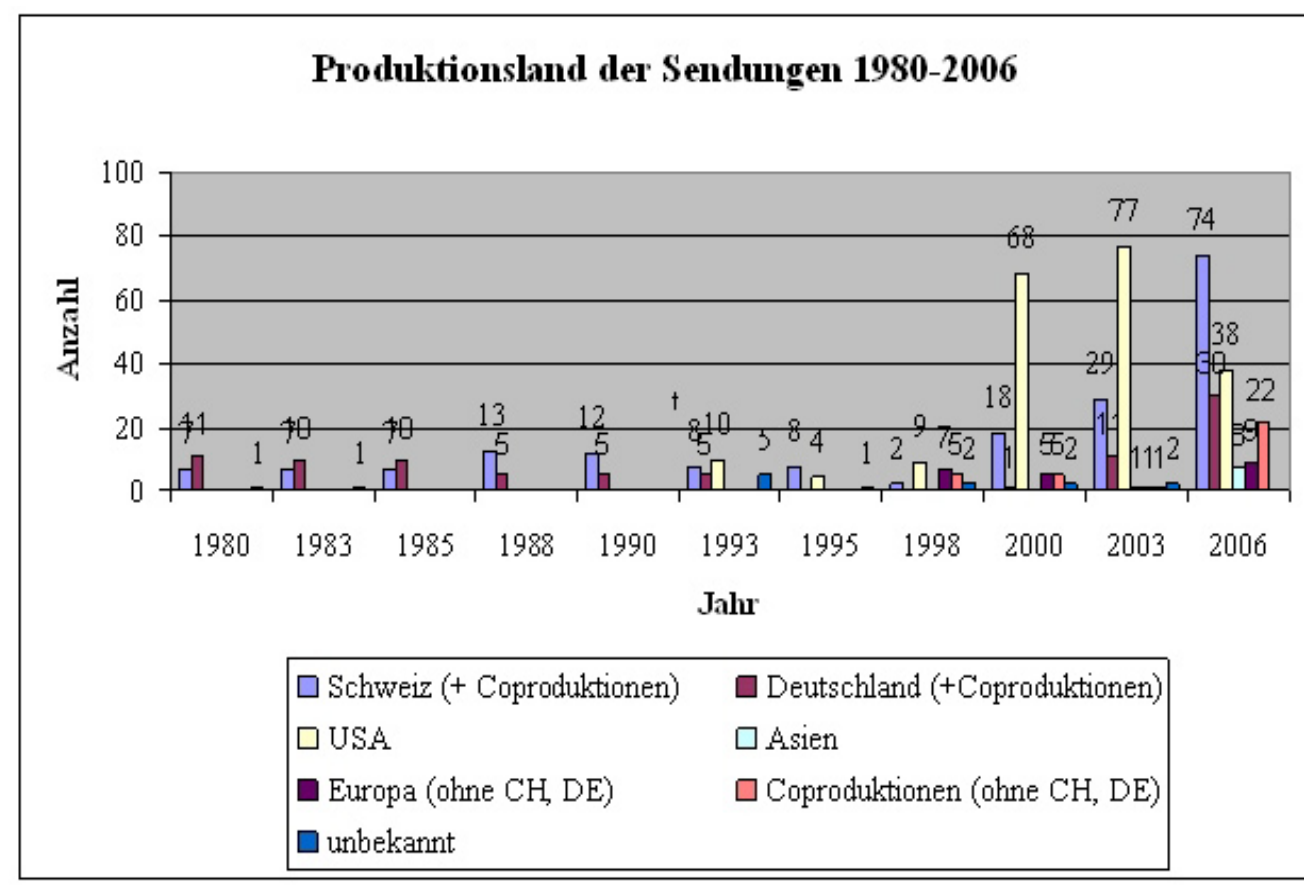

Grafik 4: Produktionsland der SF-Kindersendungen von 1980-2006 ( $n=582)$ 


\subsubsection{Genres der SF-Kindersendungen 1980-2006}

Codiert wurden insgesamt 14 verschiedene Genres (Dokumentation/Portrait, Vorschulsendungen, Show allgemein, Spiel-/Quizshow, Bastelshow, Kochshow, Film allgemein, Soap, Comedy, Abenteuersendung, Märchen, Mystery/Horror, Zeichentrick/Animation, Magazin), welche meist nur vereinzelt und in unregelmässigen Zeitabständen vorkommen. Detailliert dargestellt werden folgend das Märchen, die Spiel-/Quizshow und der Zeichentrick/Animation.

Das Märchen ist eines der beliebtesten Genres, welches zwar eine lange Karriere aufweist, aber heute nur noch selten vorkommt. Dagegen boomt der Zeichentrick seit Mitte der 90er Jahre enorm mit steigender Tendenz, ebenso bei den Spiel- und Quizshows, allerdings in geringerem Ausmass als beim Zeichentrick (siehe Grafik 5).

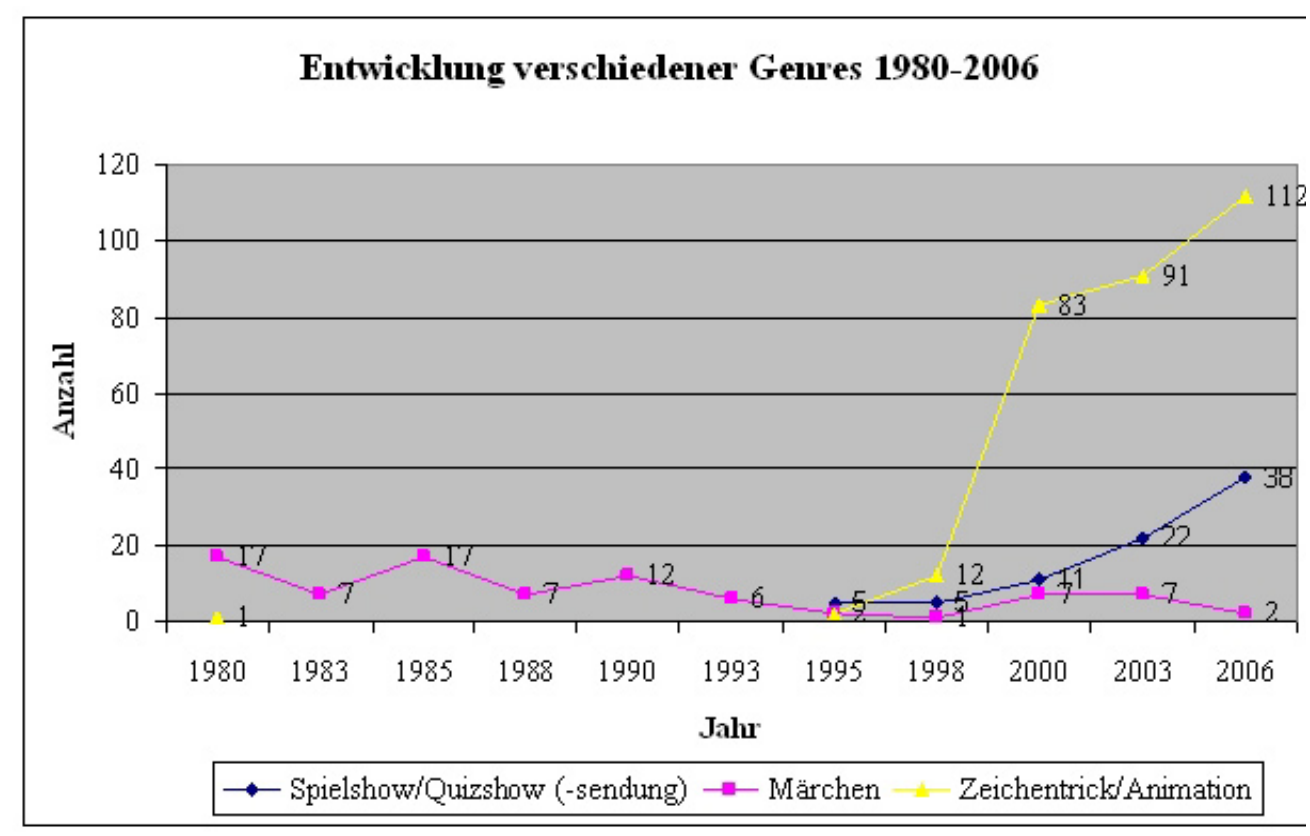

Grafik 5: Entwicklung ausgewählter Genres im SF-Kinderprogramm ( $n=583)$

\subsubsection{Das Bildungspotential der SF-Kindersendungen 1980-2006}

Der Begriff Bildung wird nicht nur in der Öffentlichkeit, sondern auch in der Wissenschaft unterschiedlich definiert und kontrovers diskutiert. Entsprechend hat die Definition von Bildung einen Einfluss darauf, ob viele Kindersendungen als bildend bezeichnet werden oder eben nicht. Dominant bildende Kindersendungen sind im SF-Kinderangebot gemäss unserer Operationalisierung praktisch keine vorhanden (siehe Grafik 6), während implizite bildende Sendungen, welche dem Infotainment zugeordnet werden können, seit dem Jahr 1998 
zunehmen. Am häufigsten vertreten, aber auch starken Schwankungen unterworfen, sind die unterhaltenden Kindersendungen. Bis 1993 bleiben sie auf relativ konstantem Niveau und halten sich mit implizit bildenden Angeboten in etwa die Waage. Ab 1998 steigen die unterhaltenden Angebote stark an, aber auch die implizit bildenden nehmen stark zu, was mit dem populären Format der Spiel- und Quizshows zusammenhängen dürfte.

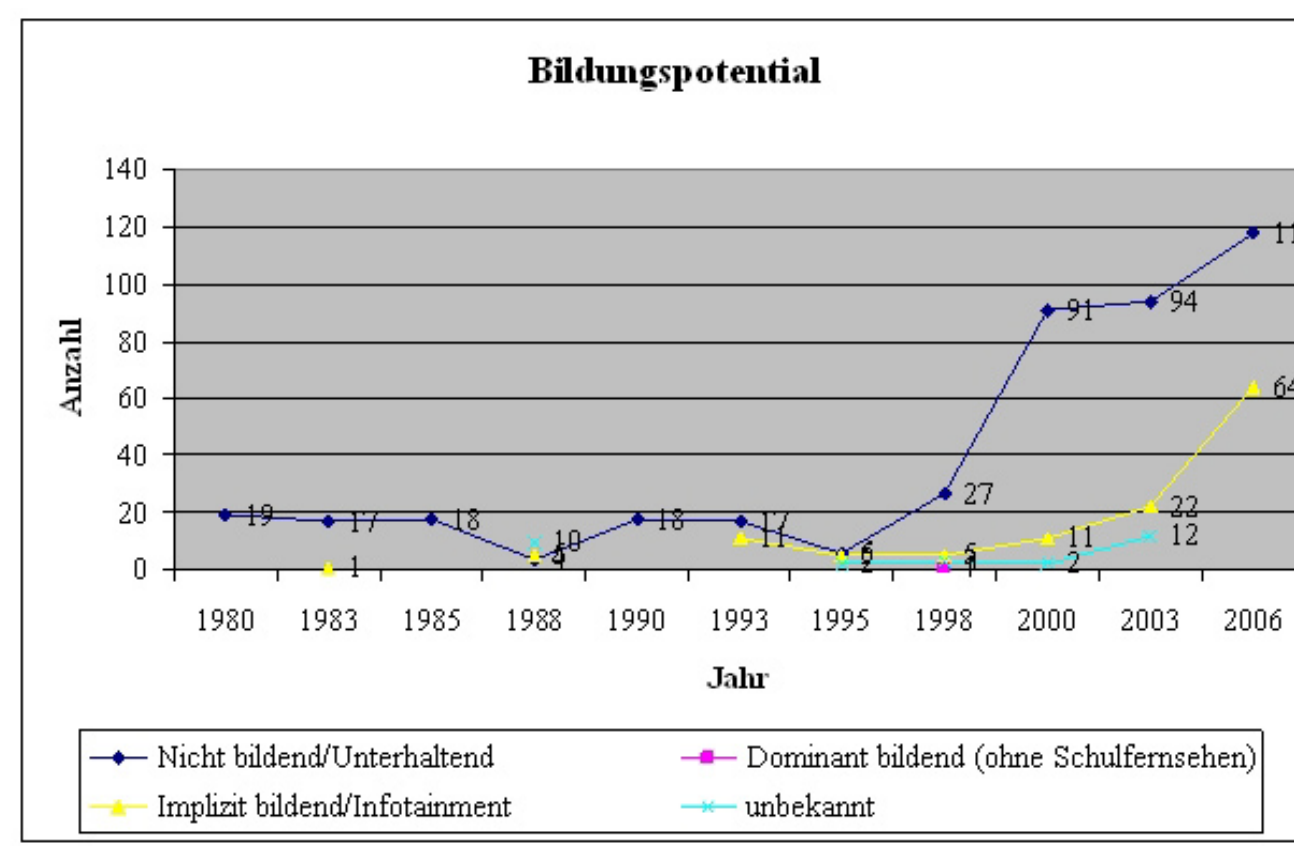

Grafik 6: Bildungspotential der SF-Kindersendungen 1980-2006 ( $n=583)$

Krüger unterteilt das Kinderprogramm 1) in konzeptionelle Kindersendungen mit pädagogischer Intention, 2) nonfiktionale oder 3. gemischte Formen. In Deutschland haben die öffentlich-rechtlichen Sender (ARD/ZDF) einen fast gleich hohen Anteil an konzeptionellen Kindersendungen wie an anderen Kindersendungen (vgl. Krüger 2005: 197), dies verhält sich im Schweizer Kinderfernsehen deutlich anders. Würde man die implizit bildenden und bildenden Kindersendungen zu Sendungen mit pädagogischer Intention zählen, sind nur halb so viele aller Kindersendungen dieser Kategorie zuzuordnen.

Wie die Untersuchung von Bachmair und Hofmann zeigt, sind Programme mit Lernorientierung bei den Kindern durchaus beliebt wie beispielsweise Die Sendung mit der Maus oder Löwenzahn (vgl. Bachmair/Hofmann 1998: 5,8). Demzufolge wäre an einem Festhalten am heutigen Ungleichgewicht zwischen nicht bildenden und bildenden Sendung aus Angst vor tiefen Einschaltquoten 
künftig abzuraten, denn auch gute bildende Sendungen können durchaus Einschaltquoten generieren.

Weiss und Trebbe kommen zum Schluss, dass bei den deutschen Kanälen nur ARD und ZDF nicht ausschliesslich unterhaltende Kindersendungen anbieten. Ein Drittel des ARD-Programms zählt nicht zu unterhaltenden Sendungen und ca. ein Viertel des Kinderprogramms des ZDF (vgl. Weiss 2000: 73); im Schweizer Kinderprogramm zählt immerhin die Hälfte des Angebots zumindest zum Typus der implizit bildenden Sendungen.

\section{Ausgewählte Ergebnisse der Expertenbefragung}

3.1 Vorgehen und Methode

Ziel der Expertenbefragung war es, retrospektiv und zukunftsorientiert eine Einschätzung der Entwicklung, der Qualität und der Funktionen des SF-Kinderangebots seit 1980 zu erhalten. Um ein möglichst differenziertes Bild der Entwicklung des Angebots und dessen Hintergründe einerseits und der Wahrnehmung des Angebots in der Öffentlichkeit andererseits entwerfen zu können, wurde die Befragung 1) aus einer Binnenperspektive und 2) aus einer Fremdperspektive angegangen. In der Binnenperspektive wurden sieben Personen befragt, welche in den Jahren 1980, 1990 und gegenwärtig für die Kinder- und Jugendangebote von SF zuständig waren bzw. sind. Es interessiert im Zeitverlauf, wie, unter welchen Bedingungen und mit welchen Perspektiven der Bildungsauftrag SF-intern definiert und umgesetzt worden ist und künftig umgesetzt werden soll. Unter der Fremdperspektive wurden fünf Personen aus dem Bildungs-, Medien- und Kulturbereich befragt, welche die Entwicklung des Kinderfernsehens im Laufe der letzten 25 Jahre kritisch mitverfolgt haben. Es interessierte, wie diese Experten die Inhalte und die Qualität des Angebots im Zeitverlauf beurteilen, welche Anforderungen sie im Rahmen des Bildungsauftrags an die SRG stellen und inwiefern sie diesen erfüllt sehen. Zudem wurde zukunftsorientiert nach den Problemen und Perspektiven im Schweizer Kinderfernsehen und bei der Erfüllung des Bildungsauftrags gefragt.

Da es auch darum ging, Veränderungen und Entwicklungen zu erfassen, wurden Personen gesucht, die das Kinderangebot über eine möglichst lange Zeitspanne prägten oder beobachteten. Die leitfadengestützten Interviews fanden im Jahre 2006 und zwar von August bis November statt. Die Auswertung der Interviews orientierte sich an der qualitativen Inhaltsanalyse nach Mayring (1999). Wegleitende Fragen für die Auswertung waren:

\section{Gegenwart:}

- Welche Zielsetzungen werden im Kinderangebot von SF verfolgt?

- Wie wird das aktuelle Angebot beurteilt?

- Der Bildungsauftrag - wie wird er definiert? 
- Das aktuelle Angebot im Lichte des Bildungsauftrags

\section{Vergangenheit:}

- Wichtigste Veränderungen im Kinderangebot von SF und auslösende Faktoren. Bewertung der Veränderungen und Folgen für die Umsetzung des Bildungsauftrags

\section{Zukunft:}

- Perspektiven, Handlungsbedarf, Trends

Im hier vorliegenden Beitrag kann nicht auf alle ausgewerteten Aspekte eingegangen werden. Wir konzentrieren uns auf die Gegenwart und damit auf die Beurteilung des aktuellen Angebots gerade auch im Hinblick auf den Bildungsauftrag.

\subsection{Ergebnisse Gegenwart Kinderprogramm SF}

\subsubsection{Welche Zielsetzungen verfolgen die Macher/innen?}

«SF will das Zielpublikum zielgruppengerecht, altersgemäss und auf festen und stabilen Sendeplätzen mit unterhaltenden und informativen Angeboten bedienen» (MTV2). Ergänzend und die genannten Aspekte gewichtend waren auf einer inhaltlichen Ebene zum Zeitpunkt der Befragung folgende Aspekte wichtig: «Wir wollen ein qualitativ anspruchsvolles Kinderprogramm. Aber wir haben nicht unbedingt einen pädagogischen Anspruch. Die Qualität unseres Kinderprogramms muss sich daran messen, ob es uns gelingt, die Ansprüche der Kinder an ein unterhaltsames Kinderprogramm zu erfüllen» (MTV1). Der Anspruch, ein qualitativ gutes, unterhaltsames Programm zu machen, korrespondiert mit Kriterien, welche z. B. bei der Auswahl von eingekauften Serien beigezogen werden. So sollen Geschichten aus der Perspektive der Kinder erzählt werden und zur Identifikation einladen. Zudem will das Kinderprogramm aktivierend sein und interaktiv. Insgesamt will SF nicht nur ein eingekauftes Programm aussenden, sondern man soll dem Programm anmerken, dass es ein Schweizer Programm ist.

Auf einer strategischen Ebene werden Marktresonanz und Markführerschaft als Zielsetzungen angegeben: SF will in der Schweiz der führende Kindersender sein: «Das Ziel ist ganz klar: Wir wollen in der Deutschschweiz der führende Kindersender werden. Das ist im Moment nicht der Fall. SuperRTL hat die besseren Marktanteile. Allerdings fehlen derzeit noch wichtige Voraussetzungen, um ganz vorne dabei zu sein. Wir senden nicht in der Kinder-Primetime zwischen 17 und 19 Uhr, und wir brauchen mehr Stabilität und Verlässlichkeit bei den Sendeplätzen» (MTV1). 
Wie der letzte Punkt zeigt, sind die genannten Zielsetzungen beim Fernsehen auf die Zukunft ausgerichtet. Das heisst, die Zielsetzungen gelten u. a. bezogen auf die Sendeplätze oder auf die Marktführerschaft zum Zeit der Untersuchung als solche nicht erfüllt.

\subsubsection{Wie wird das aktuelle Angebot beurteilt?}

Die befragten Personen (Beobachter/innen, frühere Macher/innen) betrachten das aktuelle Angebot mehrheitlich kritisch. Als Stärken gelten folgende Aspekte. Sie wurden allerdings jeweils nur von einzelnen Personen genannt:

- Es wird ein Bemühen um inhaltliche Vielfalt festgestellt.

- Die Sendungen sind mehrheitlich verständlich und bieten vereinzelt Ansätze von Interaktivität.

- Es wird ein sorgfältiger Umgang bezüglich Aspekten wie Gewaltlosigkeit, Gender, Altersrelevanz, Sprache, Moral und Menschenbild festgestellt.

- Einige Trickfilme sind synchronisiert.

- Das Kinderfernsehen hat einen relativ guten Bekanntheitsgrad.

Insgesamt überwiegt allerdings die Kritik am aktuellen Angebot:

- Zu viele Sendungen werden eingekauft. Gemäss einem Macher beträgt der Anteil an Eigenproduktionen für einen normalen Wochentag ein Achtel des Gesamtangebots. Bei einem knappen weiteren Achtel handelt es sich um schweizerdeutsch synchronisierte Programme.

Als Probleme bei eingekauften Sendungen gelten folgende Punkte:

- Auf eingekaufte Angebote kann inhaltlich nicht mehr Einfluss genommen werden. Die konzeptuelle Leistung der Redaktion beschränkt sich auf die Auswahl der auf dem Markt vorhandenen (mehr oder weniger guten) Produkte.

- Die eingekauften Sendungen stammen meistens aus anderen Kulturkreisen. Das Produkt ist nicht in der lokalen Kultur verankert, in welcher Kinder aufwachsen, spezifisch Schweizerisches kann nicht einfliessen.

- Die Sendeplätze sind ungünstig und nicht stabil. Das Kinderangebot wird zurzeit zu Zeitpunkten (vor 17 Uhr) ausgestrahlt, die von der Zielgruppe kaum genutzt werden können. Zudem wurde das Kinderangebot in der jüngeren Vergangenheit immer wieder verschoben. Bei sportlichen Grossereignissen wie die Fussball WM, welche hohe Einschaltquoten generieren, fällt das Kinderangebot, teilweise über Wochen, ersatzlos aus.

- Eigenproduktionen: «Die selber gemachten Sachen sind nicht schlecht, sie sind einfach viel zu kurz» (B6). Die Eigenproduktionen werden zudem inhaltlich als wenig anspruchsvoll eingestuft: "Es gibt kaum [wirkliche/us] Eigenleistung. Ich erwarte von einer Kindersendung, dass recherchiert und gefilmt 
wird, eine Reportage entsteht, oder ein Gespräch dazu. Ich möchte Inhalt und nicht einfach die Zeit füllen mit Telefongesprächen und Spielchen» (B7).

- Die Zielgruppe gilt als zu weit gefasst bzw. aufgrund der Fokussierung auf das Primarschulalter (7 bis 12 Jahre) zu wenig differenziert angesprochen: Zudem fehlt ein spezifisches (schweizerisches) Vorschulprogramm. Auch die Zielgruppe der Teenager wird heute - anders als noch in den 1990er-Jahren - nicht mehr bedient.

- Zwei Beobachter/innen sprechen von einem Abbau der Moderationen und der zunehmenden Vermarktung von Produkten ohne unterbrechende Moderation unmittelbar vor und nach Trickfilmen oder Serien.

Ein Beobachter moniert zusammenfassend: «Vergleicht man heute mit der grossen Zeit des Kinderfernsehens mit Sesamstrasse, Rappelkiste, Pusteblume und in der Schweiz Spielhaus, so steht das Angebot heute kläglich da» (B3).

\subsubsection{Der Bildungsauftrag}

Alle Befragten wurden gebeten zu definieren, was sie unter dem Bildungsauftrag verstehen. Generell ist zu bemerken, dass die Vorstellungen darüber, was Bildung bzw. der Bildungsauftrag meint, innerhalb der Befragten sehr individuell und vom eigenen persönlichen und beruflichen Hintergrund geprägt sind: Folgende Aspekte des Bildungsauftrags liessen sich - nicht immer trennscharf - eruieren:

- Bildung als (Sach)Informations- oder Wissensvermittlung

- Bildung als Meta-Wissen und Reflexionsfähigkeit und als Teilaspekt: Bildung als Medienbildung

- Bildung als Wertvermittlung und Gemütsbildung

- Bildung als Vermittlung von Sprache und wertvollen Kulturgütern

Bezogen auf die Zielgruppe der Kinder realisiert sich der Bildungsbegriff immerhin bei einer Mehrheit der Befragten in einer Art Vermittlung eines umfassenden Orientierungswissens bis hin zur Persönlichkeitsbildung. In diesem Verständnis wird davon ausgegangen, dass die Situation des Heranwachsens eine besondere ist, in welcher es neben der kognitiven auch emotionale und soziale Förderung braucht. Die Heranwachsenden werden dabei als offene und in ihrer Entwicklung zu unterstützende oder begleitende Persönlichkeiten betrachtet.

Unter Bildung subsumiere ich alles, was den Kindern und Jugendlichen hilft, in ihrer Welt, Umwelt und Gesellschaft zurecht zu kommen, all das sind eigentlich bildende Inhalte (MTV4).

Wenn ich auf meine Zielgruppe schaue, besteht der Bildungsauftrag darin, den Kindern ihre Welt auf ihre Art und Weise zu erschliessen und 
dass sie kognitiv in die Lage versetzt werden, unabhängiger zu handeln (MTV1).

Die verschiedenen Aussagen verweisen darauf, dass Bildung bezogen auf Kinder über das Vermitteln von Sachwissen hinausgeht und in einem engen Bezug zur Sozialisation, Enkulturation und zum Selbstständig- und Mündigwerden gesehen wird. Ein Befragter erwähnt hier explizit den folgenden Aspekt: Bildung ist prozesshaft und wird ausgelöst durch die Konfrontation und Auseinandersetzung mit Themen und Inhalten, was auch von Rundfunkmedien geleistet werden kann.

\subsubsection{Wird im Kinderprogramm ein Bildungsauftrag erfüllt?}

Die gegenwärtigen Produzenten/-innen vom SF-Kinderangebot bekennen sich explizit zu einem Unterhaltungsauftrag.

Das Schulfernsehen leistet einen Beitrag, um den Bildungsauftrag bei einem jüngeren Publikum zu erfüllen. Das Kinderprogramm hat nicht explizit die Erfüllung dieses Auftrags im engeren Sinn zum Ziel. Wir haben jedoch ganz klar und primär einen Unterhaltungsauftrag (MTV1).

Trotz der Distanznahme zum Bildungsauftrag sehen die Macher/innen in ihrem Fernsehprogrammangebot einzelne Elemente, über welche eine bildende Funktion wahrgenommen wird und wahrgenommen werden könnte.

[Der Bildungsauftrag/us], das ist ein Wort, das wir so in unserem Alltagsgebrauch nicht verwenden. Wir diskutieren allerdings: Was für moralische Werte hat ein Programm, was für Inhalte und Verhaltensformen werden vermittelt? Was für eine Art von Humor ist da? (MTV2).

Während SF zum Befragungszeitpunkt ein nicht auf Bildung zielendes Programm ausstrahlt, wird im Ausland ein Trend hin zu edukativen Programmen für Kinder z. B. in den Bereichen Ernährung, Gesundheitserziehung, Sprachenlernen festgestellt.

Frühere Macher/innen und Beobachter/innen sind mehrheitlich der Meinung, dass das SF-Kinderfernsehen einen Bildungsauftrag erfüllen sollte. Sie erkennen jedoch im zum Untersuchungszeitpunkt ausgestrahlten Programmangebot kaum bildende Elemente. Sofern der Bildungsbegriff z. B. als unterhaltendes WissensQuiz gefasst ist (1, 2 oder 3), gibt es Ansätze dafür. Sobald der Bildungsbegriff jedoch als Hinführung an gesellschaftlich wichtige, für Kinder anregende, aktuelle oder brisante Themen wie etwa Krieg, Religion, Sexualität oder auf Werte oder Aspekte des sozialen Zusammenlebens ausgeweitet wird, fällt das Urteil 
negativ aus. Im Gegensatz zu einem früheren Macher, welcher den Bildungsauftrag für das Medium Fernsehen generell als nicht mehr zeitgemäss betrachtet, weil das Fernsehen zu additiv und zu wenig interaktiv sei, sehen die Beobachter jedoch durchaus Möglichkeiten und vor allem eine Verantwortung darin, dass ein öffentlicher Sender auch noch zum heutigen Zeitpunkt aufgrund eines Qualitätsbewusstseins inspirierende und anregende Programme für Kinder ausstrahlt oder produziert:

Ich bilde mir ein, dass es möglich ist Bildungsfernsehen zu machen, mit echten Kindern, wo man echt Themen anspricht, die durchaus zum Beispiel Kindernachrichten sein könnten [...]. Man kann auch Bildungsinhalte in hübsche kleine Geschichten verpacken, es muss nicht immer alles lustig, lässig, seltsam, fremd und abgedreht sein. Für mich ist wirklich was vom Wichtigsten das Nachvollziehen können, lebensecht sein, lebendig im Sinn von richtige Menschen und richtige Situationen zeigen (B6).

Das Ziel wäre, anhand von Geschichten in unterhaltender Form [und als Eigenproduktion/us], Beispiele für erwünschtes Sozialverhalten zu vermitteln. Es wäre das Ziel, Kindern zu helfen, sich in ihrer Umgebung zurecht zu finden ... da gehört natürlich die Familie, die Schule, die Freunde/-Innen [dazu/us]. ... Das wäre für mich schon ein Ziel, wo das Fernsehen einen Beitrag leisten könnte, wenn Kinder schon fern schauen. Im kognitiven Bereich denke ich kann das Fernsehen [auch/us] etwas vermitteln ... (B5). Spannende Reportagen, Einblicke in andere Welten [...]. Auch Ratgebersendungen, die gibt es ja für Erwachsene: Gesundheitssendungen, ,Konsum', Wirtschaftssendungen, politische Sendungen. Für Kinder sollte es Ähnliches geben: Was mache ich, wenn ich Probleme habe mit meinen Eltern oder Streit mit dem Bruder oder Zoff in der Schule? SF könnte doch sicher irgendeine gute Sendung produzieren (B7).

Als wichtig erachtet wird zusammenfassend, dass ein bildendes Kinderfernsehen einen Bezug zur Realität des zusehenden Kindes herstellt und Betroffenheit, Interesse und schliesslich Prozesse auslöst, die als kognitiv und emotionale anregend, inspirierend und in diesem Sinne bildend betrachtet werden können. Dies setzt nach Meinung einiger Befragten qualitativ hoch stehende Produktionen und vor allem mehr Eigenproduktionen voraus, in welchen Orientierung, Affinitäten zur lokalen Kultur, zur hiesigen Lebenswelt der Kinder und eventuell zum hiesigen Bildungssystem möglich sind. An die Macher/innen wird der Anspruch erhoben, dass sie ein hohes Mass an Kreativität aufweisen, kreativen Freiraum haben und wissen, wie Kinder verschiedenen Alters angesprochen werden können und wie sich auch bildende Inhalte attraktiv umsetzen lassen. 
Aufgrund der durchwegs kritischen Position der Beobachter/innen, stellt sich die Frage nach den Faktoren, welche das Angebot für Kinder im Laufe der letzten rund 25 Jahren prägten. Wie kommt es dazu, dass der Bildungsaspekt bei den Machern/-innen wenig zählt, das SF-Kinderprogramm im Gesamtprogramm wenig Konstanz aufweist und mehrheitlich eingekauft wird? Als wichtigste Faktoren gelten die Dominanz ökonomischer Faktoren (Quoten, Werbung etc.), seit der Dualisierung im Fernsehbereich, aber auch unternehmensinterne Faktoren: So wurde die für das Kinderprogramm zuständige Abteilung «Familie und Bildung» 1993 aufgelöst und das Kinderprogramm zuerst in die Abteilung «Film, Serien und Jugend» und 2004 in die Abteilung "Unterhaltung» integriert, was die inhaltliche Gewichtung deutlich vorgibt. Wegen seiner geringen marktwirtschaftlichen Ausstrahlung verlor das Kinderprogramm zudem intern an Stellenwert und wurde immer wieder Opfer von Sparmassnahmen. Dies führte (bis heute) zum Abbau von Stellenprozenten und Manpower in der Redaktion, zum Abbau von Eigenproduktionen und zum fast vollständigen Abbau von Sendungen vor allem für Jugendliche und Kinder im Vorschulalter. Die Entwicklungen im Rundfunkbereich wie auch das strategische Verhalten von SF werden in einem engen Zusammenhang zur gegenwärtigen Situation gesehen:

Im [gegenwärtigen] Kinderfernsehen sehe ich den Bildungsauftrag nicht erfüllt [...]. Es darf keinen Quotendruck geben auf ein Kinderprogramm [...]. Ich finde ganz schrecklich, dass der Service Public (SF) eigentlich versucht hat - in seiner Angst, Kunden an das Privatfernsehen zu verlieren - dauernd und immer noch das Privatfernsehen [...] zu kopieren. Billiger, schneller und unüberlegter zu produzieren, wegzusparen [...] statt sich auf die eigenen Stärken zu besinnen ... [und zu sagen/us] wir haben Subventionsgelder und könnten mit denen was Anständiges machen (B6).

Die nicht allein im Hinblick auf den Bildungsauftrag wenig befriedigende Entwicklung des Kinderprogramms im Schweizer Fernsehen sowie dessen Bedeutungsverlust innerhalb der Organisation, sind gemäss einem Beobachter auch auf der Folie eins gesellschaftlichen und ideologischen Wandels und eines Bedeutungsverlusts von Kindern in der Öffentlichkeit seit den 1970er Jahren zu sehen. So wird auch von mehreren Befragten erwähnt, dass Kinder bzw. das Kinderprogramm in der Schweiz keine Lobby mehr hätten.

Die Zukunft des Schweizer Kinderfernsehens wird aus Sicht aller Befragten in einen engen Zusammenhang gebracht mit stabilen Sendeplätzen und mehr Ressourcen. Mehr Ressourcen müssten bedeuten, dass mehr Zeit zur Verfügung stände für Analysen, die Entwicklung von Konzepten sowie für den Aufbau von Fachkompetenz. Allerdings ist die gegenwärtige Situation unberechenbar. Just zum Befragungszeitpunkt waren die gegenwärtigen Macher/innen mit der An- 
kündigung einer Neukonzeption des Kinderprogramms konfrontiert und u.a. mit einem Stellenabbau. Pläne, das Kinderprogramm auf einem Kinderkanal, evtl. in Kooperation mit dem KIKA auszustrahlen, sind zurzeit aus finanziellen Gründen (im Zusammenhang mit dem neuen RTVG) auf Eis gelegt. Auch auf der geplanten Wissensplattform des SF sind keine expliziten Angebote für Kinder vorgesehen. Insofern ist nicht damit zu rechnen, dass sich unter den gegenwärtigen Rahmenbedingungen in naher Zukunft etwas verändern wird.

\section{Fazit}

Im vorliegenden Beitrag wurde die Entwicklung des Kinderprogramms im Schweizer Fernsehen im Hinblick auf den Bildungsauftrag mit zwei verschiedenen Methoden nachgezeichnet. Folgendes, grobes inhaltliches Fazit kann gezogen werden: Die quantitative Programmanalyse weist nach, dass das Kinderangebot im Schweizer Fernsehen seit 1980 absolut zugenommen, im Verhältnis zum gesamten SF-Angebot aber etwas abgenommen hat, meist die Darstellungsform und das Genre des Zeichentricks wählt und heute zu fast zwei Dritteln unterhaltende bzw. nicht bildende Sendungen ausstrahlt. Diese Verhältnisse entsprechen im Vergleich mit Deutschland mehr dem Angebot der privaten Fernsehsender als jenem der öffentlich-rechtlichen Anbieter. Die befragten Experten bestätigen den grossen Anteil an eingekauften Sendungen bei einem kontinuierlichen Abbau von Eigenproduktionen. Zudem wird eine ausgesprochenen Unterhaltungsorientierung mit dem Ziel, Einschaltquoten und damit Markterfolg zu generieren, festgestellt. Insgesamt wird ein kritisches Bild vom "Kinderfernsehen Schweiz» gezeichnet. So wird deutlich, dass das Kinderprogramm im Schweizer Fernsehen inhaltlich und konzeptionell nicht den Erwartungen der befragten, externen Beobachtern/-innen an ein öffentliches, qualitativ anspruchsvolles, auch bildendes Kinderprogramm entspricht. Unter der Perspektive der befragten Machern/-innen ist der Druck des Umfeldes spürbar, sprich: interne und externe Konkurrenz sowie schwierige institutionelle Rahmenbedingungen (Programmplätze, Ressourcen, Stellenwert der Kinderprogrammredaktion im Unternehmen). Diese prägen das Schaffen und die Zielsetzungen (Unterhaltungs- und Marktorientierung) massgeblich.

Diese Befunde sind, befragt man die Kinderprogrammgeschichte, nicht überraschend und lassen sich mit den von Erlinger (1998) formulierten Entwicklungslinien für das Kinderfernsehen in Deutschland in Verbindung bringen: Nach Erlinger ist das Kinderfernsehen der 1990er-Jahre u. a. geprägt von mehr Programm - d. h. vor allem mehr Animation und Trickfilm, mehr Kommerz und mehr Strategie, bei weniger Identifizierbarkeit und Relevanz (vgl. Erlinger 1998: 5, 7, 478), was für die Schweiz durchaus bestätigt werden kann. Parallelen gibt es auch zur Feststellung, dass sich das Kinderfernsehen um deutschen Raum inhaltlich nicht mehr weiterentwickle, denn entscheidend ist nach Erlinger heute nicht, «welche 
Programme Kinder ,brauchen', entscheidend für Bestand und Entwicklung des Kinderprogramms sind die ... ermittelten Zuschauerzahlen» (Erlinger 1998: 5). «Kein Anbieter kann sich momentan ein nachfrageschwaches Kinderprogramm leisten» (Erlinger 1998: 5). Auch diese Ausrichtung lässt sich für die Schweiz als Grundtenor bestätigen, wobei die Situation für das Schweizer Kinderprogramm insofern eine besonders angespannte ist, als dass der Schweizer Markt ein geteilter (nach Sprachregionen) und dadurch überaus begrenzter und zusätzlich vom deutschen Nachbarn stark konkurrenzierter ist. Unter diesen Bedingungen zeigen sich Ansätze zu einem ebenfalls von Erlinger in Aussicht gestellten kontinuierlichen Abbau von Kinderangeboten im Gesamtprogramm (vgl. Erlinger 1998: 5). Die eventuelle Auslagerung auf einen Kinderkanal muss für die Schweiz (wie für Deutschland) kontrovers diskutiert werden, denn die Ausgangslage gestaltet sich anders als in Deutschland. Generell würde ein eigener Schweizer Kinderkanal strukturelle Probleme, wie fixe Sendeplätze, für Kinder geeignete Sendezeiten etc. umgehend lösen. Inhaltlich muss aber einerseits eine grössere Programmfläche gestaltet werden, welche die unten genannten minimalen Anforderungen unbedingt erfüllen sollte, andererseits ist unklar mit welchen Mitteln mehr und qualitativ hochstehender Inhalt finanziert werden soll.

Auch wenn sich die Statements der befragten, externen Beobachtern/-innen unter den gegebene Realitäten und Trends nostalgisch und stark normativ geprägt anhören, stellen sie zusammen mit den Befunden der Programmanalyse die Frage in den Raum, ob es für ein Schweizer Kinderprogramm Alternativen gäbe oder solche gefordert werden müssten. Basierend auf dem Verfassungsartikel und dem Radio- und Fernsehgesetz wurden darum im Rahmen des Projektes entgegen der herrschenden Trends folgende politikorientierte Empfehlungen als minimale Anforderungen bezüglich eines Schweizer Kinderprogramms formuliert:

- Analog zu den Erwachsenen haben Kinder grundsätzlich ein Recht, neben dem massenmedialen Unterhaltungsprogramm, auf ein qualitativ anspruchsvolles und bildungsorientiertes Kinderprogramm. Die Einhaltung und Umsetzung dieses im RTVG formulierten Rechts ist nur möglich, wenn das Kinderprogramm institutionell in der Organisation des SRG stark verankert wird und zwar in Form von Redaktionen bzw. Abteilungen mit einem redaktionellen Leitbild.

- Zwar wird das Fernsehen vornehmlich als Unterhaltungsmedium und zum Spass genutzt, dennoch sollten Formate, welche Spass und Lernen verbinden, vermehrt auch relevante Interessen der Kinder aufgreifen und deren Wissensdurst stillen. Besonders zu betonen sind in diesem Zusammenhang eigenproduzierte Sendungen, welche an den Kinderalltag anknüpfen und zur kulturellen Orientierung in der Schweiz beitragen. 
- Sowohl die prioritär unterhaltenden als auch die prioritär informierenden und bildenden Angebote des Kinderprogramms sollten minimalen Qualitätsansprüchen genügen. Strukturelle Qualität verlangt ein genügend grosses und bezüglich Genres abwechslungsreiches Kinderangebot mit fixen Sendezeiten, welche dem kindlichen Lebensrhythmus angepasst sind. Inhaltliche Qualität verlangt nach für Kinder verständlichen, nachvollziehbaren Sendeinhalten mit Identifikationsmöglichkeiten prosozialer Art.

Aus einer eher methodischen Perspektive zeigte das Projekt auf, wie schwierig es ist, Daten basierend auf verschiedenen methodischen Vorgehensweisen sauber aufeinander zu beziehen. Die Programmanalyse ist eher grob und liefert wenige Kontextinformationen; die Expertenbefragung widerspiegelt subjektiven Wahrnehmungen, Erfahrungen und normative Vorstellungen. Da die Stichprobe nicht sehr gross war und die Befragten aus verschiedenen zeitlichen Abschnitten stammten, war die Generalisierung und Einordnung der Stellungsnahmen nicht immer einfach. Zudem fällt auch auf, dass die Beobachter - zum Teil inspiriert aus früheren Zeiten - stark inhaltlich-pädagogisch und aus einem Anspruch an ein öffentliches Medium heraus argumentieren, während die Macher/innen stärker Fragen der Umsetzung (formal) und der Resonanz im Publikum (Publikumszuwendungen, Einschaltquoten) gewichten. Letzteres ist auch ein Hinweis darauf, wie herausfordernd es für Kinderprogrammschaffende insgesamt sein muss, in dynamischen Medien- und Lebenswelten von heutigen Kindern überhaupt Resonanz zu erzeugen.

\section{Literatur}

Bachmair, Ben. «Bestandsaufnahme. Konzept. Forschungsprojekt.» Internationales Zentralinstitut für das Jugend- und Bildungsfernsehen (IZI) 〈http://www.br-online.de/jugend/izi/bestand/konzept.htm〉 (2.4.2007).

Bachmair, Ben/ Hoffmann, Ole. «Lernen mit dem Kinderfernsehen: Wunsch oder Wirklichkeit? » Televizion 2 (1998): 4-20.

Bonfadelli, Heinz / Schwarb, Ursula / Signer, Sara / Schade, Edzard. Öffentlicher Rundfunk und Bildung. Angebot, Nutzung und Funktionen von Kinderprogrammen. Schlussbericht zuhanden des BAKOM. Zürich, 2007.

Diekmann, Andreas. Empirische Sozialforschung. Grundlagen, Methoden, Anwendungen. 5., durchges. Aufl. Reinbek b. Hamburg: Rowohlt, 1999.

Doelker-Tobler, Verena. «Medienkundliche und medienkritische Sendungen im Fernsehrprogramm für Kinder und Jugendliche: Ein stufenübergreifendes Aktivierungskonzept.» Medienpädagogik und Kommunikationslehre Hrsg. v Georg Wodraschke. München: Ölschläger, 1979. 177-186.

Erlinger, Hans Dieter u. a. (Hg.). Handbuch des Kinderfernsehens. 2., überarb. u. erw. Aufl. Konstanz: UVK Medien, 1998.

Hohlfeld, Ralf. «Fernsehprogrammanalyse: Formen, Einsatzmöglichkeiten und Reichweite.» Fernsehforschung in Deutschland. Hrsg. v. Walter Klingler u. Gunnar Roters u. Oliver Zöllner. Baden-Baden: Nomos, 1998. 197-224. 
Krüger, Michael. «Das Problem bleibt das Problem. Eine Replik zum Beitrag von Weiss und Trebbe.» Inhaltsanalyse. Perspektiven, Probleme, Potentiale. Hrsg. v. Werner Wirth u. Edmund Lauf. Köln: Herbert von Halem, 2001. 72-81.

Krüger, Michael. «Tendenzen in den Programmen der grossen Fernsehsender 1985 bis 1995. Elf Jahr Programmanalyse im dualen Rundfunksystem.» Media Perspektive 8 (1996a): 418-440.

Krüger, Michael. «Unterschiede der Programmprofile bleiben bestehen. Programmanalyse 1996: ARD, ZDF, RTL, SAT.1 und PRO SIEBEN im Vergleich.» Media Perspektiven 7 (1996b): 354-366.

Krüger, Michael. «Sparten, Sendungsformen und Inhalte im deutschen Fernsehangebot. Programmanalyse 2004 von ARD/Das Erste, ZDF, RTL, SAT.1 und ProSieben.» Media Perspektiven 5 (2005): 190-204.

Lambrecht, Clemens. «Bestandesaufnahme zum Kinderfernsehen 2002. Programm in der Angebotsperspektive.» Bestandsaufnahme zum Kinderfernsehen 〈http://www.kinderfernsehforschung.de〉 (23.05.2006)

Loretz, Niklaus. «Forderungen an die Medienpädagogik: gesellschaftsbezogen und empanzipatorisch. Für einen Unterricht mit, über und gegen Film und Fernsehen.» ZOOM 20 (1979): 2-5.

Mayring, Philipp. Einführung in die qualitative Sozialforschung. München: Belz Studium, 1999

Portmann, Stefan. "Die Stellung der SRG in einem zeitgemässen Bildungssystem.» 39. Jahresbericht (1969):28-31.

Stalder, Hanspeter. «Medienerziehung in der Primarschule.» ZOOM 1 (1978): 2-7.

Weiss, Hans-Jürgen/Trebbe, Joachim. Fernsehen in Deutschland 19981999. Programmstrukturen, Programminhalte, Programmentwicklungen. Berlin: Verlag (Schriftenreihe der Landesmedienanstalt), 2000.

\section{Footnotes}

1 Im Verfassungsartikel 93 Radio und Fernsehen Abschnitt 2 steht: «Radio und Fernsehen tragen zur Bildung und kulturellen Entfaltung, zur freien Meinungsbildung und zur Unterhaltung bei. (...)». Im RTVG (Bundesgesetz über Radio und Fernsehen) in Kapitel 2 «Schweizerische Radio- und Fernsehgesellschaft» 1. Abschnitt «Programmauftrag und Konzession» Litera c wird von der SRG SRR «Bildung des Publikums, namentlich durch die regelmässige Ausstrahlung von Sendungen mit bildenden Inhalten» verlangt. 